\title{
Oral Intake of Specific Bioactive Collagen Peptides Reduces Skin Wrinkles and Increases Dermal Matrix Synthesis
}

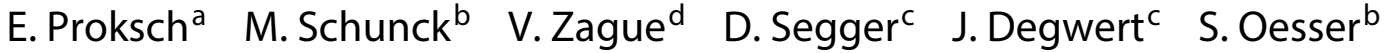 \\ ${ }^{a}$ Department of Dermatology, University of Kiel, and ${ }^{\mathrm{b}}$ Collagen Research Institute, Kiel, and ${ }^{\mathrm{C}}$ Skin Investigation and

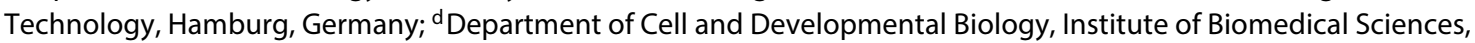 \\ University of São Paulo, São Paulo, Brazil
}

\section{Key Words}

Bioactive collagen peptide . Collagen peptides · Skin .

Wrinkles · Type I collagen · Elastin · Fibrillin

\begin{abstract}
Dietary consumption of food supplements has been found to modulate skin functions and can therefore be useful in the treatment of skin aging. However, there is only a limited number of clinical studies supporting these claims. In this double-blind, placebo-controlled study, the effectiveness of the specific bioactive collagen peptide (BCP) VERISOL ${ }^{\circledR}$ on eye wrinkle formation and stimulation of procollagen I, elastin and fibrillin biosynthesis in the skin was assessed. A hundred and fourteen women aged 45-65 years were randomized to receive $2.5 \mathrm{~g}$ of $\mathrm{BCP}$ or placebo, once daily for 8 weeks, with 57 subjects being allocated to each treatment group. Skin wrinkles were objectively measured in all subjects, before starting the treatment, after 4 and 8 weeks as well as 4 weeks after the last intake (4-week regression phase). A subgroup was established for suction blister biopsies analyzing procollagen I, elastin and fibrillin at the beginning of the treatment and after 8 weeks of intake. The ingestion of the specific BCP used in this study promoted a statistically significant reduction of eye wrinkle volume $(p<0.05)$ in com-
\end{abstract}

parison to the placebo group after 4 and 8 weeks (20\%) of intake. Moreover a positive long-lasting effect was observed 4 weeks after the last BCP administration $(p<0.05)$. Additionally, after 8 weeks of intake a statistically significantly higher content of procollagen type I (65\%) and elastin (18\%) in the BCP-treated volunteers compared to the placebo-treated patients was detected. For fibrillin, a $6 \%$ increase could be determined after $\mathrm{BCP}$ treatment compared to the placebo, but this effect failed to reach the level of statistical significance. In conclusion, our findings demonstrate that the oral intake of specific bioactive collagen peptides $\left(\right.$ Verisol ${ }^{\circledR}$ ) reduced skin wrinkles and had positive effects on dermal matrix synthesis.

(c) 2013 S. Karger AG, Basel

\section{Introduction}

Skin appearance and integrity get worse with age due to the synergistic effects of chronological and photoaging, hormonal deficiency and influences of environmental factors [1]. As a consequence of a decline in several metabolic activities, like quantitative and qualitative changes in dermal collagen and elastin, the skin constitution changes and typical symptoms of aging are visible. The loss of connective tissue in cutaneous aging results in de-

\section{KARGER}

E-Mail karger@karger.com

www.karger.com/spp
(C) 2013 S. Karger AG, Basel

$1660-5527 / 13 / 0273-0113 \$ 38.00 / 0$
Professor E. Proksch

Christian Albrecht University of Kiel, Department of Dermatology

Schittenhelmstrasse 7

DE-24105 Kiel (Germany)

E-Mail EProksch@dermatology.uni-kiel.de 
creased elasticity, loss of skin tone and a progressive deepening of facial creases and wrinkling [2].

Wrinkles on the face are the most prominent recognized signs of skin aging. Takema et al. $[3,4]$ reported an age-related decrease in skin elasticity and a coriaceous appearance of affected skin areas. Especially facial skin sites like the corners of the eyes are mostly susceptible to wrinkle formation, also popularly known as crow's feet.

Skin functions and healthy appearance depend on a sufficient supply of essential nutrients. The relationship between nutrition and skin has become a hot topic all over the world. Intervention studies indicate that it is possible to modulate or delay skin aging and to improve skin integrity by dietary ingredient supplementation [5].

In preclinical studies it was shown that collagen peptides had stimulatory effects on type I collagen and other extracellular matrix molecules in human fibroblasts $[6$, 7]. Moreover, in animal studies it was demonstrated that the density and diameter of fibroblasts as well as of collagen fibrils increased by collagen peptide administration whereas a UVB-induced reduction of type I collagen was diminished after bioactive collagen peptide (BCP) treatment $[8,9]$.

However, there is no clinical evidence that BCP supplementation may reduce wrinkle formation and benefit skin metabolism after oral intake in humans. In this study, we objectively evaluated the efficacy of a specific BCP with regard to the volume of eye wrinkles after 8 weeks of daily intake. Moreover, the influence of the BCP treatment on the content of procollagen type I, elastin and fibrillin in blister suctions was assessed. To the best of our knowledge, this is the first double-blind, placebo-controlled study showing that a dietary supplement composed of a specific collagen peptide is effective in reducing wrinkles and aging-related skin biomarkers.

\section{Material and Methods}

\section{Test Product}

The test product used in this study was a BCP composed of different specific collagen peptides with a high safety profile derived from a complex multistep procedure by degradation of porcine type I collagen. The product was provided by Gelita AG (Eberbach, Germany), commercially available as Verisol. The product is clearly defined by a matrix-assisted laser desorption ionization mass spectrometry mass peaks fingerprint with specific collagen peptides of an average molecular weight of $2.0 \mathrm{kD}$.

\section{Study Design}

The study was carried out as a monocentric, double-blind, randomized, placebo-controlled supplementation study on the effects of a specific BCP on eye wrinkle volume (primary out- come) and content of type I procollagen, elastin and fibrillin in skin fluid (secondary outcomes) after 8 weeks of daily intake.

The study was approved by the Freiburger Ethik-Kommission International, Freiburg, Germany, and adhered to current good clinical practice regulations. All test subjects received detailed information listing every single parameter relevant to the study. All subjects gave signed, informed consent after receipt of the written information and having had a possibility for further questioning.

\section{Subjects}

A total of 114 healthy female subjects, 57 subjects allocated to each treatment group, were enrolled in the study and randomized to treatment with a daily dose of $2.5 \mathrm{~g}$ of BCP or placebo (maltodextrin). Forty-eight of these (24 per treatment group) were included in the suction blister biopsies for the subsequent analysis of different skin aging-related biomarkers.

The products were taken orally by the subjects at home according to the instructions given by the investigator. The powder was to be dissolved in water or any other liquid.

Prior to the beginning of oral treatment and data acquisition there was a preconditioning period of at least 7 days. Within this period and throughout the entire period of the study, the subjects had to refrain from using any leave-on products and oily or moisturizing skin-cleansing products on the arms and the area around the eyes. Moreover, use of make-up, skincleansing tissues, wipes for make-up removal and cleansing lotion on the test sites was prohibited. It was also prohibited to intensively expose the test sites to UV light (sun or solarium). The subjects were not allowed to visit saunas or swimming pools, and to do intensive sport during the day prior to the study visits. Moreover, they were prohibited from changing their living or dietary habits, and from consuming any additional nutritional supplement or vitamin preparations 4 weeks prior to the start of the study and during the study. The treatment of the test areas with the following medication was not allowed prior to the start and during the study: dermatological therapeutics (6 weeks previously), corticosteroids and antihistamines (4 weeks previously), anti-inflammatory drugs and antibiotics (2 weeks previously).

\section{Inclusion Criteria}

The inclusion criteria were as follows: healthy females ranging in age from 45 to 65 years (homogeneous distribution between treatment groups), phototypes I-III (Fitzpatrick scale), in general good health and mental condition, personal informed consents to participate in the study, personal presence on the predefined days at the institute, willing and able to follow the study rules and a fixed schedule.

\section{Exclusion Criteria}

The exclusion criteria were as follows: any deviation from the above-mentioned inclusion criteria, acute skin diseases (e.g. atopic eczema, atopic dermatitis, psoriasis) on the test sites, or other dermatological disorders (scars, sunburn, moles), food allergies against ingredients of the test products, gastrointestinal diseases or indigestion, tattoos on the test sites, topical medication used in the test area within 6 weeks prior to the start of the study, systemic medication with anti-inflammatory agents or antibiotics within 2 weeks prior to the start, systemic medica- 
tion with corticoids and/or antihistamines within 4 weeks prior to the start, medication with an anticoagulant, proneness to hyperpigmentation or hypertrophic scar formation, other systemic medication within 4 weeks prior to the start, systemic illness of the subject at the beginning of the study, pregnancy or a period of breast feeding, immunological disorders, severe disorders within 6 months prior to the start, e.g. cancer, acute cardiac and circulatory disorders, severe diabetes, alcohol and drug abuse, participation in other studies with cosmetic products in the test areas within 2 weeks prior to the start or during the study, participation in a study with a pharmaceutical preparation within 4 weeks prior to the start, intake of nutritional supplements within 4 weeks prior to the start and, except for the test products administered during the study, change in lifestyle or eating habits during the study, treatment with leave-on products, oily or moisturizing skin-cleansing products on the arms, or a change in the usual skin care routine, exposure to intensive sunlight or artificial UV (solarium) light on the test sites within 1 week prior to the start or during the study, swimming, sauna or intensive sport within 1 day prior to measurements, lack of compliance, intellectual or mental inability to follow study instructions.

\section{Assessments}

Test Sites

The test sites were the wrinkle area around the left eye (lateral canthus) for eye wrinkle measurements and the inner aspect of the right forearm for the suction blister biopsies used for the subsequent analysis of procollagen type I, elastin and fibrillin.

On every measurement day, the subjects had to expose their uncovered test sites to the indoor climatic conditions $\left(21.5^{\circ} \mathrm{C} ; 50 \%\right.$ relative humidity) for at least $30 \mathrm{~min}$.

\section{Measurement Times}

There were 4 measurement times for eye wrinkle assessments: immediately before starting the product treatment $\left(\mathrm{t}_{0}\right)$, after $4\left(\mathrm{t}_{1}\right)$ and 8 weeks $\left(\mathrm{t}_{2}\right)$ of daily product intake, and 4 weeks after the last intake ( $t_{3}, 4$-week regression phase).

Suction blisters were generated before starting the treatment $\left(\mathrm{t}_{0}\right)$ and after 8 weeks $\left(\mathrm{t}_{2}\right)$ of intake.

In each case the subject's compliance (dosage and way of intake) and tolerance towards the products were checked after 1, 4 and again after 6 weeks of intake.

\section{In vivo Measurement of the Eye Wrinkle Volume}

The influence of BCP on the eye wrinkle volume was measured at the outer corner of the eye (lateral canthus) using the optical 3 -dimensional in vivo measuring instrument Primos ${ }^{\circledR}$ Compact (GF-Messtechnik GmbH). Three measurements were conducted per test site. The size of the measurement area was $30 \times 40 \mathrm{~mm}^{2}$. The postbaseline measurements were performed using the overlay function. For each subject, the original pictures of the reference files at the baseline and the corresponding measurement files of the postbaseline measurements were brought into congruence using the 3-dimensional matching function. Height images were computed according to the standard procedure using mathematical filters. These height images were used to calculate the eye wrinkle volumes (in cubic millimeters of one selected wrinkle). After manual marking of the selected eye wrinkles, the volume was computed by the Primos software. This was performed for all 3 images that were taken per test site and measurement point in time. Then, the mean of the 3 single measurements was calculated for each test site and point in time.

\section{Suction Blister Biopsies}

To quantify the amount of procollagen I, elastin and fibrillin in the skin of the volunteers before and after treatment with BCP, the so-called suction blister model was applied, according to Kiistala [10]. Per point in time, 2 suction blisters of $7 \mathrm{~mm}$ in diameter were generated in the test area. To induce the blisters, Plexiglas suction chambers with 2 circular openings each with a diameter of $7 \mathrm{~mm}$ were placed on the test site. A vacuum pressure of about $450-850$ mbar was applied. The blisters were induced within $1.5-2.5 \mathrm{~h}$. The liquids of all suction blisters were collected using sterile hypodermic syringes. The liquids were pooled, collected in cryovials at $-20^{\circ} \mathrm{C}$ and stored frozen at $-80^{\circ} \mathrm{C}$ immediately after preparation. The small wounds were covered with a wound occlusive and healed completely and scar free within 6-10 days. On completion of the study, the blister fluids were used for the quantitative analysis of procollagen type I, elastin and fibrillin.

Enzyme-Linked Immunosorbent Assays for the Analysis of Procollagen Type I, Elastin and Fibrillin

In vitro enzyme immunoassay kits were used for the quantitative analysis of human procollagen type I (Takara Bio Inc., Japan), human elastin (Cusabio Biotech, China) and human fibrillin-1 (Cusabio Biotech) in the suction blister fluids.

The tests were performed according to the respective instruction manual. Briefly, samples were diluted 1:25 vol/vol with kit sample buffers to reach concentrations within the range of the standards. Every suction blister sample was measured in duplicate. Moreover, the recovery of the enzyme immunoassay kits was analyzed, revealing recovery rates of more than $80 \%$ on average.

\section{Statistical Analysis}

All data were tested for normal distribution by the 1-sample Kolmogorov-Smirnov test. Statistically significant differences between both treatment groups were analyzed by the 2 -sided independent samples $t$ test. The hypothesis of a normal distribution was accepted when there was a $\mathrm{p}$ value $>0.05$. As for the differences between the treatment situations, for a $p$ value $<0.05$ the difference was accepted as statistically significant.

The following treatment situations for wrinkle volume changes were compared: treatment situations at points in time $t_{0}$ (baseline situation), $t_{1}$ and $t_{2}$ (after 4 weeks and 8 weeks of treatment) and $t_{3}$ (4-week washout phase).

The results obtained from the blister suction fluids were expressed in relation to the initial situation and pairwise differences were tested for statistical significance between both treatments.

\section{Results}

\section{Subjects and Dropouts}

The results of 114 subjects were involved in data analysis. The subjects were between 45.0 and 65.4 years old $(55.6 \pm 6.0)$, without statistically significant differences between the treatment group and the placebo group 
(table $1 ; \mathrm{p}=0.998$ ). A hundred and eight of the 114 subjects finished the study correctly and completely for the eye wrinkle measurements. There were 6 dropouts, none related to the product intake or the study procedure in general.

As for analysis of the suction blister fluids, 40 of the 48 subjects (20 per group) finished the study correctly and completely. The 40 subjects were between 45.8 and 65.0 years of age $(55.9 \pm 6)$. There were 8 dropouts, none related to the product intake or the study procedure in general.

No treatment side effects were observed in any of the volunteers.

\section{Eye Wrinkle Volume}

At the beginning of the study the volume of eye wrinkles between both treatment groups were not statistically significantly different $\left(0.381 \pm 0.36\right.$ vs. $0.375 \pm 0.26 \mathrm{~mm}^{3}$, $\mathrm{p}=0.93)$.

After 4 weeks of treatment the BCP group showed a statistically significantly reduced eye wrinkle volume of more than $7.2 \%$ in comparison to the placebo group ( $\mathrm{p}<$ 0.05 ). This positive effect was more pronounced after 8 weeks of intake. At this point in time wrinkle volume in the BCP group was significantly $(\mathrm{p}<0.01)$ reduced by $20.1 \%$ on average $\left(0.326 \pm 0.38\right.$ vs. $\left.0.408 \pm 0.25 \mathrm{~mm}^{3}\right)$ compared to the placebo group (fig. 1a, b). In particular a maximum reduction in eye wrinkle volume of $49.9 \%$ was achieved.

Four weeks after the last product intake (4-week regression phase), the BCP treatment group still showed a statistically significant decrease in eye wrinkle volume of $11.5 \%(\mathrm{p}<0.01)$, as shown in figure 2. It is notable that in the active agent group at the end of the regression phase the same decreased mean eye wrinkle volume $(0.326$ $\mathrm{mm}^{3}$ ) was determined as at the end of the treatment period after 8 weeks.

\section{Blister Suction Fluids}

Due to inhomogeneous data at the beginning of the investigation (prior to treatment) between the drug and the placebo groups the results of procollagen, elastin and fibrillin measurements were expressed in relation to baseline data $\left(\mathrm{t}_{0}\right)$.

\section{Procollagen Content}

Procollagen type I content was increased by $65 \%$ after 8 weeks of BCP treatment compared to the placebo group. This pronounced impact on collagen synthesis was statistically significant $(\mathrm{p}<0.01$; fig. 3 ).
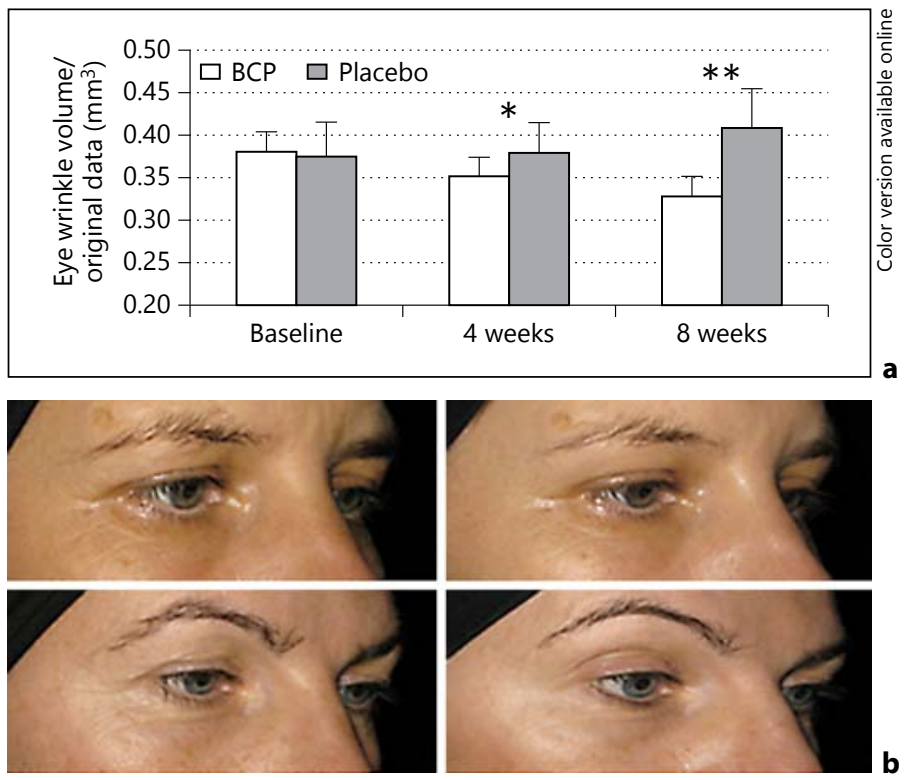

Fig. 1. a Orally administered BCP led to a statistically significant reduction of eye wrinkle volume after 4 and 8 weeks of treatment. Over the same period of time, eye wrinkle volume in the placebotreated group increased continuously (mean \pm SEM; $\mathrm{n}=57$; ${ }^{*} \mathrm{p}<$ $\left.0.05,{ }^{* *} \mathrm{p}<0.01\right)$. b Visible reduction of eye wrinkle volume after 8 weeks of BCP intake. Exemplary pictures of 2 participants of the active agent group before (left) and after (right) treatment.

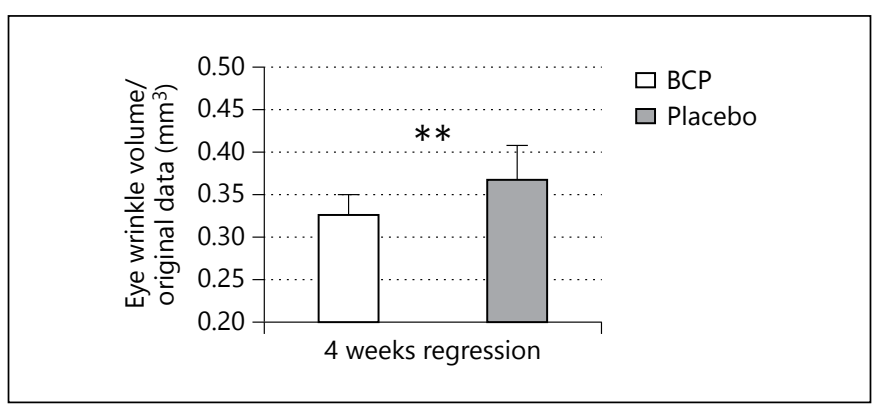

Fig. 2. Reduction of eye wrinkle volume persisted in BCP-treated volunteers 4 weeks after last intake (mean \pm SEM; $\mathrm{n}=57$; ${ }^{* *} \mathrm{p}<$ $0.01)$.

Table 1. Demographic data on the study subjects

Group Baseline $\left(t_{0}\right) \quad 4$ weeks $\left(t_{1}\right) \quad 8$ weeks $\left(t_{2}\right) \quad$ 4-week regression $\left(t_{3}\right)$

\section{Subject number}

$\begin{array}{lllll}\text { A } & 57 & 55 & 55 & 52 \\ \text { B } & 57 & 55 & 55 & 55\end{array}$

Mean age $\pm \mathrm{SD}$, years

A $\quad 55.6 \pm 5.7 \quad 55.4 \pm 5.7 \quad 55.4 \pm 5.7 \quad 55.4 \pm 5.5$

B $\quad 55.6 \pm 6.2 \quad 55.6 \pm 6.3 \quad 55.6 \pm 6.3 \quad 55.6 \pm 6.3$




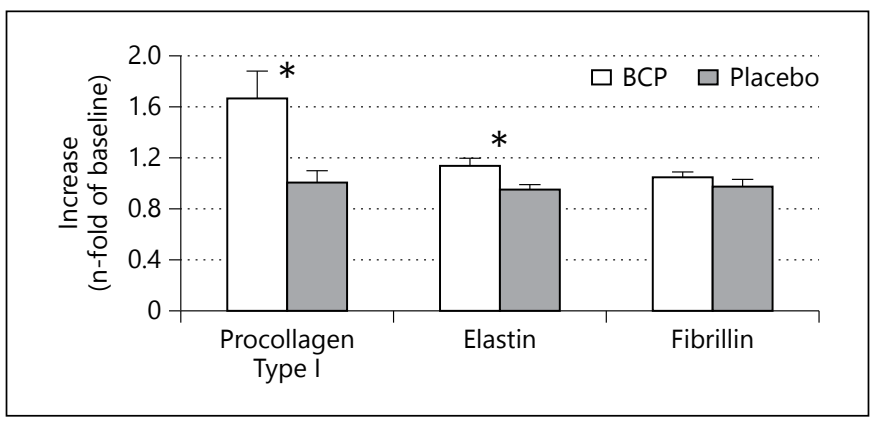

Fig. 3. The amount of procollagen type I and elastin was statistically significantly increased 8 weeks after BCP administration, in contrast to placebo treatment. The content of fibrillin in suction blister fluid was increased after 8 weeks of BCP ingestion by trend (mean \pm SEM; $\mathrm{n}=20 ;^{*} \mathrm{p}<0.05$ ).

\section{Elastin Content}

In addition to procollagen, $\mathrm{BCP}$ intake also promoted a statistically significant $(\mathrm{p}<0.01)$ increase in elastin content of $18 \%$ in comparison to the placebo treatment after 8 weeks (fig. 3 ).

\section{Fibrillin Content}

Ingestion of BCP led to a clear increase in fibrillin content of $6 \%$ in comparison to placebo after 8 weeks of treatment but this result failed to reach the level of statistical significance (fig. 3).

\section{Discussion}

Throughout our life skin integrity changes and shows a decrease in elasticity and moisture, as well as a noticeable increase in wrinkles. Skin wrinkle formation is the most prominent sign of growing old $[1,2]$ and seems to be more common in the skin of Caucasian people [11]. In a prospective study it was found that wrinkle onset was delayed by about 10 years in Chinese women compared with French women [12].

Independently of wrinkle incidence and skin ethnic aspects, concerns about changes in physical appearance brought on by aging are very common. As the demand for interventions to ameliorate visible signs of aging is continuously growing [13], interest in the development of dietary supplements and functional food products for skin health has increased as well.

In preclinical and clinical studies food supplements have been considered as effective cosmeceutical substances with a potential for reducing wrinkles and improving skin appearance. Especially the efficacy of orally administered collagen peptides on skin health has been demonstrated in several investigations [14-17].

The current study is the first investigating the efficacy of a specific, orally administered BCP $\left(\right.$ Verisol $^{\circledR}$ ) on eye wrinkles, demonstrating a statistically significant reduction of eye wrinkle deepness (crow's feet) in comparison to a placebo treatment. At the end of the treatment after 8 weeks the eye wrinkle volume in the verum group had decreased by $17.7 \%$ compared to the baseline, whereas in the placebo group in the same time period the wrinkle volume had increased by $14.5 \%(\Delta 32.2 \%)$.

The observed changes in the placebo group might have been caused by altered weather and climatic conditions during the study period. It is most unlikely that the determined effect is caused by diet-related alterations, as the subjects were instructed not to modify their lifestyle during the study period. In addition, a direct impact of the placebo can be excluded as no influence of maltodextrin on skin physiology has been described in the literature. Therefore it can be assumed that the variations in the placebo group do not refer to systematic effects.

Apart from the observed efficacy of an oral Verisol ${ }^{\circledR}$ treatment, it is striking that the positive effect on skin health was persistent for at least 4 weeks after having stopped the BCP intake. A possible explanation for this long-lasting improvement might be the pronounced increase in the biosynthesis of essential dermal macromolecules such as collagen, elastin and fibrillin. It is known that collagen and elastin are the major components in the dermis supporting preservation of skin structure, skin firmness and elasticity, and that they directly affect wrinkle formation. In contrast to the healthy skin situation, an alteration of elastic fibers and degradation of collagen bundles does occur in the dermis of wrinkled skin. It is known that collagen fiber deficiencies in aged and photoaged human skin are a major cause of skin wrinkling.

Apart from the visible changes in skin wrinkles, in the current study the synthesis of these dermal extracellular matrix proteins which are crucial for skin health and wellbeing was investigated in suction blister fluids. It was demonstrated that at the end of the treatment period the collagen content was increased 1.65-fold and that the amount of elastin was increased 1.2-fold after Verisol treatment, compared to the placebo group.

In addition to these positive impacts the ingestion of BCPs led to an increase of $6 \%$ in the content of fibrillin in comparison to placebo, by the end of the study period. Fibrillins are the most important components of microfibrils and are necessary for the integrity of elastic fiber bundles described by Lee et al. [15], who observed a re- 
duced fibrillin-1 expression in wrinkled skin, which demonstrated the important role of fibrillin for skin functionality. Fibrillin connects elastic fibers with small leucinerich proteoglycans (like decorin and biglycan) which are essential for the water-binding capacity in connective tissues [18].

Overall, BCP treatment seems to have a positive impact on important dermal macromolecules [19] which have a direct influence on skin wrinkle formation. Our current findings confirm previous reports from experimental and animal trials demonstrating the stimulatory effects of collagen peptides on anabolic processes supporting the maintenance of the dermis extracellular matrix $[6-9,20]$. Our in vitro studies on primary human dermal fibroblasts demonstrated a stimulatory effect of Verisol $^{\circledR}$ on the expression of skin extracellular matrix macromolecules. After supplementation of the specific collagen peptides we were able to demonstrate a pronounced, statistically significant increase in type I collagen expression as well as proteoglycan expression, such as biglycan, decorin and versican (data not published).

Apart from these positive influences of Verisol ${ }^{\circledR}$ for dermal matrix synthesis it has been described that the daily intake of collagen peptides decreases expression levels of matrix metalloproteinase-2, a catabolic enzyme which is, for instance, responsible for collagen type IV breakdown [7].

Type IV collagen forms a highly cross-linked network essential for mechanical stability of the basement membrane and is therefore an important factor for skin wrinkles and furrow formation [21]. The observed stimulatory impact on the dermal matrix of specific BCPs, as well as the reported anticatabolic effect, suggest a possible mechanism for explaining the significant wrinkle reduction demonstrated by the Verisol ${ }^{\circledR}$ treatment.
Another factor that promotes wrinkle formation is decreased skin elasticity, as shown by Fujimura et al. [22]. Skin elasticity is influenced by several parameters, like elastic fiber formation and skin moisture. In a previous study we investigated the effects of daily Verisol ${ }^{\circledR}$ ingestion in 35- to 55-year-old female volunteers on skin elasticity and other skin wrinkle-related parameters. In a double-blind, randomized, placebo-controlled clinical study, skin elasticity was significantly improved in women who received $2.5 \mathrm{~g} /$ day of a specific BCP for 8 weeks. In addition, the results revealed that the observed positive effects of BCP administration were more pronounced in women who were over 50 years old, as indicated by increased skin hydration and improved skin elasticity [23].

\section{Conclusions}

Based on the results of the study, it can be concluded that the oral ingestion of specific collagen peptides led to a pronounced, statistically significant reduction of eye wrinkle volume. In contrast to most topically applied substances this positive effect on the skin seems to be caused by a direct impact on dermal extracellular matrix turnover, as demonstrated by a significant increase in collagen and elastin synthesis. The direct effect on the dermal matrix might explain the long-lasting improvement of skin wrinkles for at least 4 weeks after the end of the supplementation of the collagen peptides. It has to be pointed out that the results presented are only valid for the specific collagen peptide composition (Verisol ${ }^{\circledR}$ ) used in this study. Other collagen hydrolysates or collagen peptides might exhibit dissenting effects. Here further research especially into the mode of action of BCPs on dermal structures would be desirable.

\section{References}

1 Imokawa G: Recent advances in characterizing biological mechanisms underlying UVinduced wrinkles: a pivotal role of fibroblastderived elastase. Arch Dermatol Res 2008; 300(suppl 1):S7-S20.

$\checkmark 2$ Calleja-Agius J, Muscat-Baron Y, Brincat MP: Skin ageing. Menopause Int 2007;13:60-64.

-3 Takema Y, Yorimoto Y, Kawai M, Imokawa G: Age-related changes in the elastic properties and thickness of human facial skin. Br J Dermatol 1994;131:641-648.

4 Takema Y, Yorimoto Y, Kawai M: The relationship between age-related changes in the physical properties and development of wrin- kles in human facial skin. J Soc Cosmet Chem 1995;46:163-173

5 Boelsma E, Hendriks HF, Roza L: Nutritional skin care: health effects of micronutrients and fatty acids. Am J Clin Nutr 2001;73:853-864.

6 Liang J, Pei X, Zhang Z, Wang N, Wang J, Li $\mathrm{Y}$ : The protective effects of long-term oral administration of marine collagen hydrolysate from chum salmon on collagen matrix homeostasis in the chronological aged skin of Sprague-Dawley male rats. J Food Sci 2010; 75:H230-H238.

7 Zague V, de Freitas V, da Costa RM, de Castro GA, Jaeger RG, Hado-Santelli GM: Collagen hydrolysate intake increases skin collagen expression and suppresses matrix metalloproteinase 2 activity. J Med Food 2011;14:618624.

8 Matsuda N, Koyama Y, Hosaka Y, Ueda H, Watanabe T, Araya T, Irie S, Takehana K: Effects of ingestion of collagen peptide on collagen fibrils and glycosaminoglycans in the dermis. J Nutr Sci Vitaminol (Tokyo) 2006; 52:211-215.

9 Tanaka M, Koyama Y-I, Nomura Y: Effects of collagen peptide ingestion on UV-B-induced skin damage. Biosci Biotechnol Biochem 2009;73:930-932. 
10 Kiistala U: Suction blister device for separation of viable epidermis from dermis. J Invest Dermatol 1968;50:129-137.

11 Green AC: Premature ageing of the skin in a Queensland population. Med J Aust 1991; 155:473-478.

12 Nouveau-Richard S, Yang Z, Mac-Mary S, Li L, Bastien P, Tardy I, Bouillon C, Humbert P, de Lacharrière O: Skin ageing: a comparison between Chinese and European populations. A pilot study. J Dermatol Sci 2005;40:187193.

13 Manriquez JJ, Majerson Grinberg D, Nicklas Diaz C: Wrinkles. Clin Evid 2008;12:1-42.

$\checkmark 14$ Bauza E, Oberto G, Berghi A, Dal CF, Domloge N: Collagen-like peptide exhibits a remarkable antiwrinkle effect on the skin when topically applied: in vivo study. Int J Tissue React 2004;26:105-111.
15 Lee J-H, Seo J-H, Park Y-H, Lim K-M, Lee S-J: The effect of hydroxyproline and Pro-Hyp dipeptide on UV-damaged skin of hairless mice. Korean J Food Sci Technol 2008;40: 436-442.

16 Kawaguchi T, Nanbu PN, Kurokawa M: Distribution of prolylhydroxyproline and its metabolites after oral administration in rats. Biol Pharm Bull 2012;35:422-427.

17 Chai HJ, Li JH, Huang HN, Li TL, Chan YL, Shiau CY, Wu CJ: Effects of sizes and conformations of fish-scale collagen peptides on facial skin qualities and transdermal penetration efficiency. J Biomed Biotechnol 2010; 2010:757301.

18 Frantz C, Stewart KM, Weaver VM: The extracellular matrix at a glance. J Cell Sci 2010; 123:4195-4200.

19 Cho S, Won CH, Lee DH, Lee MJ, Lee S, So SH, Lee SK, Koo BS, Kim NM, Chung JH: Red ginseng root extract mixed with torilus fructus and corni fructus improves facial wrinkles and increases type I procollagen synthesis in human skin: a randomized, double-blind, placebo-controlled study. J Med Food 2009; 12:1252-1259.
20 Zhang Z, Wang J, Ding Y, Dai X, Li Y: Oral administration of marine collagen peptides from chum salmon skin enhances cutaneous wound healing and angiogenesis in rats. J Sci Food Agric 2011;91:2173-2179.

21 Contet-Audonneau JL, Jeanmaire C, Pauly G: A histological study of human wrinkle structures: comparison between sun-exposed areas of the face, with or without wrinkles, and sun-protected areas. Br J Dermatol 1999;140:1038-1047.

22 Fujimura T, Haketa K, Hotta M, Kitahara T: Loss of skin elasticity precedes to rapid increase of wrinkle levels. J Dermatol Sci 2007; 47:233-239.

23 Proksch E, Segger D, Degwert J, Schunck M, Zague V, Oesser S: Oral supplementation of specific collagen peptides has beneficial effects on human skin physiology: a doubleblind, placebo-controlled study. Skin Pharmacol Physiol 2014;27:47-55. 